\title{
THE GGS SPACECRAFT, SOLAR ARRAYS FOR SPACE PHYSICS
}

\author{
Thomas Spitzer \\ NASAGSFC, Space Power Applications Branch, Code 734, Greenbelt, MD 20771
}

\begin{abstract}
Two Global Geospace Science (GGS) spacecraft, WIND and POLAR, support highly sensitive instruments for measurements in the plasma source and plasma storage regions of geospace. The increased sensitivity of the electric and magnetic field instruments on the GGS missions required NASA Goddard Space Flight Center and Lockheed Martin Astro Space (LMAS) to explore new and innovative methods of providing an electrostatically clean, equipotential surface that would minimize interference with the surrounding plasma environment.

This paper will summarize the qualification program, test results, flight build, and on orbit performance of the GGS solar arrays.

\section{INTRODUCTION}

Both GGS spacecraft are spin stabilized $2.4 \mathrm{~m}$ diameter by $1.8 \mathrm{~m}$ high cylinders with numerous deployable booms and body mounted solar arrays. Cylindrical side array area for WIND and POLAR is approximately 9.5 square meters, while POLAR supports an additional 2.5 square meter solar array area on one end to accommodate beta angles of up to 70 degrees. Earlier conductive equipotential solar arrays have been flown on missions such as the International Sun Earth Explorer ISEE [1] and also will be flown on the upcoming Fast Auroral Snapshot (FAST) mission [2].

Important requirements were to minimize stray magnetic fields and power ripple generated by the spinning laboratories. Magnetic cleanliness was achieved by backwiring each of the 750 cell strings to cancel the effects of current generated fields. Cell layout was carefully adjusted to limit power ripple to plus or minus 5\% of the spin average. A resistor was also placed across each string isolation diode to reduce the voltage difference between lit and unlit strings.
\end{abstract}

\section{THE QUALIFICATION BOARD PROGRAM}

Three solar array Qualification Boards (QBoards) were designed, built and tested. Each board used a unique method to present an electrostatically clean surface to the surrounding environment. Two of the boards also shielded the normally exposed intercell contacts to provide an equipotential surface. Primary requirements were that the surface resistivity of the solar array would be less than $480 \mathrm{k}$ ohms/square and that an equipotential surface of less than $1 \mathrm{~V}$ would be maintained. Any design used would have minimal effect on cell efficiency and none on circuit performance. Weight and manufacturability were secondary drivers. After being built the Q-Boards were subjected to vibration testing, thermal cycling to the flight profile, and extensive electrical testing. A Q-Board qualification report was written and one design chosen for use on the flight arrays.

\section{Q-Board Description}

Three Q-Boards were built and tested between July 1990 and February 1991. The basic construction of these boards involved standard techniques. Two strings of 64 silicon Back Surface Field Reflector (BSFR) $2 \times 2$ $\mathrm{cm}$ solar cells were bonded to each of three pieces of 30 $X 30 \times 2.54 \mathrm{~cm}$ thick honeycomb substrate that conformed to the 2.4 m diameter curvature of the spacecraft. Cell sub modules had previously been built up and glassed with .10 $\mathrm{cm}$ Indium Tin Oxide (ITO) coated fused silica coverglasses. Backside components were common to all three boards and consisted of two core grounding patches, a Kapton shunt dissipater, temperature sensors, terminal board, diode board, connector, bypass diodes, and Z-306 paint with tacked wires. Three coverglass interconnect schemes were then implemented on these Q-Boards.

The GGS1 approach used thin pieces of silver mesh cell interconnect material as the grounding and shielding method. $2 \mathrm{~cm}$ long pieces of this material were placed over the gap between each cell in the cell strings. Longer pieces of this same material were formed into channels and placed into the space between the strings themselves. Coverglasses on this board featured small solder pads in each corner that had previously been hand tinned onto the ITO coating. The entire grid of silver mesh and coverglasses was then soldered together at the tinned points to form a continuous conductive surface, effectively a Faraday shield. This surface was then returned to ground through \#30AWG wires soldered to a face sheet core ground. Solar cell circuit terminations were then insulated with Kapton tape and all outer surfaces of the QBoard covered with a perforated aluminum tape. The perforations allowed the substrate to breathe and help prevent tape delamination during thermal cycling.

The GGS2 approach was identical to GGS1 except that the coverglasses were not tinned and the entire surface was tied together using CV1500 conductive RTV instead of solder.

The GGS3 approach was most similar to the previously flown ISEE array. The conductive coverglasses were tied together with a simple \#30AWG silver wire bonded with conductive Eccobond $57 \mathrm{C}$ epoxy. Since intercell gaps were not shielded on this board it did not meet primary science requirements and was considered a backup in case all else failed. A bead of insulating RTV 
142 was also placed on each cell interconnect strain relief loop on this board. Pieces of silver mesh rather than \#24AWG wire were used for the sub module interconnects and turn-arounds to save weight, ease manufacturing and reduce shadowing.

As expected the GGS3 design had the least effect on output. Effects increase with increasing angle on GGS1 and GGS2 with GGS1 showing the most degraded output.

\section{GGS Solar Array Design Selection}

The results of the Q-Board test program clearly support the use of CV1500 conductive RTV and silver mesh as the method of providing the equipotential conductive surface properties required by the GGS laboratories. Surface resistance was well within the requirement of $480 \mathrm{~K}$ ohms/square and the silver mesh provides shielding of the intercell potentials. Power output was very close to predictions. The final weight was within margins and though all three designs involve tedious, labor intensive methods this design was the most easily produced.

In addition two features proved out on the GGS3 $Q$-Board will be incorporated into the design. A bead of insulating RTV 142 will be placed over each intercell contact to prevent shorting to coverglass interconnects. Silver mesh, rather than 24AWG wire will also be used on the flight panel string turn-arounds.

\section{THE FLIGHT ARRAYS}

The WIND design features include:

$9.5 \mathrm{sq}$. meter active area

12 side panels

23,616 cells total

64 series by 369 parallel strings

470 max. Beginning of Life (BOL) $28 \mathrm{~V}$

$370 \mathrm{~W}$ min. End of Life (EOL) @ 28V

The POLAR design features include:

12 sq. meter active area

12 side panels

2 end panels

30,232 cells total

65 series by 361 parallel strings - side panels

101 series by 67 parallel strings -end panels

$565 \mathrm{~W}$ max. BOL $@ 28 \mathrm{~V} @ 135$ degrees sun angle

350W min.EOL@28V@90 degree sun angle

\section{Flight Panel Construction}

The GGS solar arrays use $2 \times 2 \times .02 \mathrm{~cm}$ thick, $n$-on-p silicon, $10 \mathrm{ohm}, B S F R$ cells and $.10 \mathrm{~cm}$ fused silica coverglasses featuring sun-side $\mathrm{MgF}_{2}$ anti-reflective and ITO conductive coatings and a cell-side UV filter coating with a 350 nanometer cut-on wavelength.

LMAS provided the panel substrates and completing all the back panel work was the first step in the build. Since GGS uses a dissipative power system, shunt resistors in the form of 10 Kapton and foil heaters were bonded to the back of each panel. Installation of temperature sensors, connectors, and panel wiring followed. The magnetic field produced by each string was minimized by running its current in a reverse direction through wires mounted to the back of the panel.

The coverglass interconnection and grounding was the most tedious aspect of the solar panel construction. Insulating RTV was applied to each of the thousands of cell interconnects to prevent them shorting to the strip of shielding mesh over each junction. Channels formed out of mesh were placed between cell strings. Then all the cells and the grid of mesh were carefully tied together with beads of CV1500 conductive epoxy. Great care had to be taken to insure that each coverglass was tied to another by at least 2 points while minimizing shadowing on the cell. Isolation tests were performed between the cell strings and substrate core ground as sections of each panel were completed to detect any shorts that may have occurred. Shielding of string potentials at the panel edges also had to be performed. This involved gluing a layer of Kapton over the string terminations followed by aluminum tape.

\section{Flight Panel Test Program}

An extensive flight panel test program was implemented throughout the build cycle to insure that both the power generating and unique electrostatic characteristics of the arrays would be preserved. Cleanliness during construction was also a major consideration since cleaning of the completed panels would be difficult. The mesh coverglass interconnects are easily snagged and any such damage would have to be reworked.

Once the basic panel construction was complete the pre-coverglass testing was done to provide baseline performance measurements. Performance tests repeated after each stage of environmental testing were compared to the initial performance as well as predictions derived from the Q-Board program. The testing performed included ground continuity and insulation resistance, wire isolation and continuity, illumination and electrical performance, thermovision, coverglass electrical continuity, thermal cycling, acoustic and vacuum bake-out.

\section{Post Spacecrait Integration}

Upon completion of spacecraft testing the panels were deintegrated, refurbished, and given an acceptance test. Refurbishment consisted of replacement of any broken cells as well as touching up the electrostatic shielding. Electrical components and wiring were checked and a full flash test at both the string and panel level performed. A $100 \%$ test of coverglass continuity was made by checking the resistance between each of the 60,000 coverglasses to ground. 


\section{SOLAR ARRAY ON - ORBIT PERFORMANCE}

The WIND spacecraft was launched in November of 1994. The spin average output of the arrays after all deployment was $437 \mathrm{~W}$, slightly higher than the specification. Ripple due to spin has been about $15 \%$ rather than the anticipated $10 \%$. This has had no effect on mission science objectives however. After more than a year in orbit output is within $2 \%$ of predicted having degraded approximately $6 \%$ since launch (see Fig. 2).

POLAR was launched in February of 1996. Its output was very close to predictions. As with WIND, ripple was higher than estimated due to the complex shadow patterns produced by the many deployables but once again this is not expected to affect science.
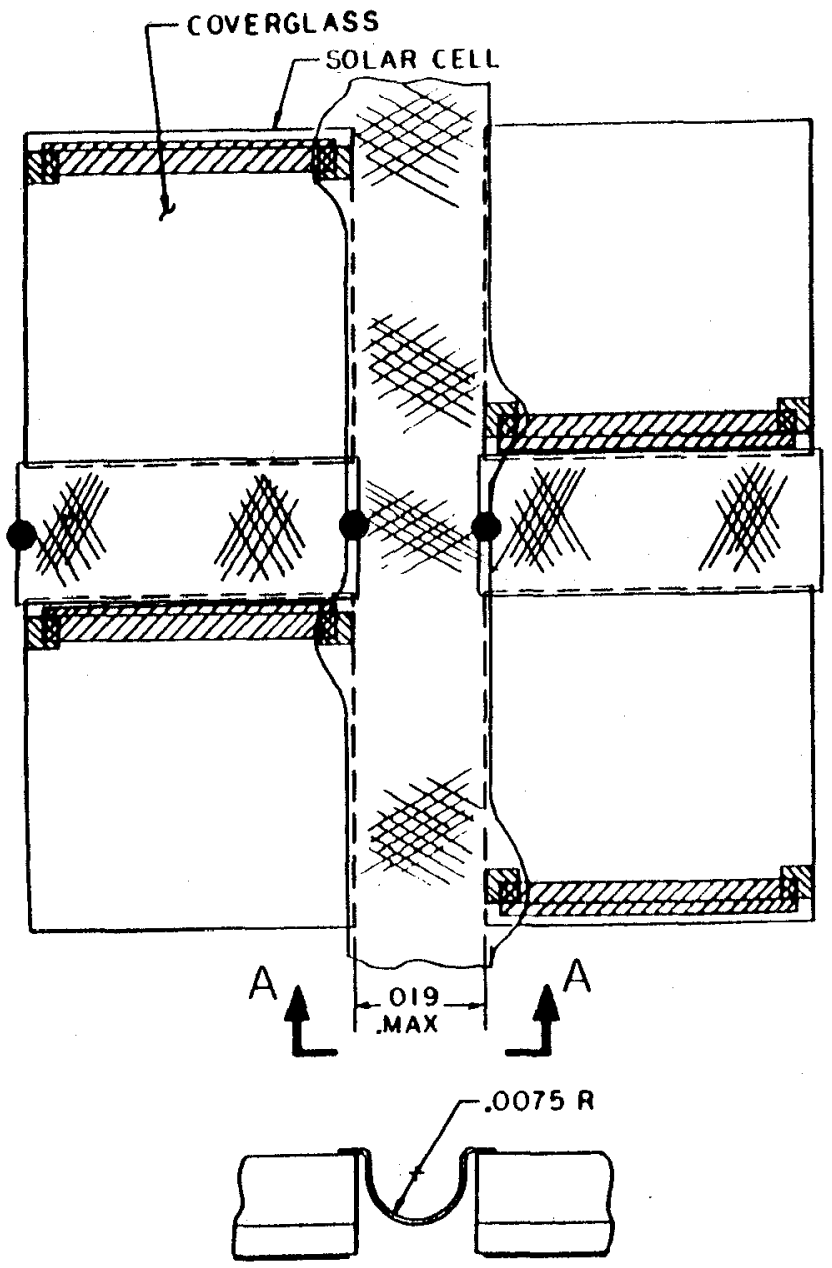

VIEW A-A

Fig. 1a GGS1. Coverglass grounding approach using silver mesh soldered to ITO coating.
Qualitatively, the mission scientists are very satisfied with WIND and POLAR performance. There is very little change in space craft charging potential while traversing diverse regions of space.

\section{REFERENCES}

[1] E. Gaddy, and J. Bass, "Interconnecting Conductively Coated Coverslides", Thirteenth IEEE PVSC, 1978, pp. 313.

[2] M. Kruer, and J. Lyons, "The FAST Solar Array: Challenging Requirements, Novel Design", Twenty-Fourth IEEE PVSC Vol. II, 1994, pp. 1949.
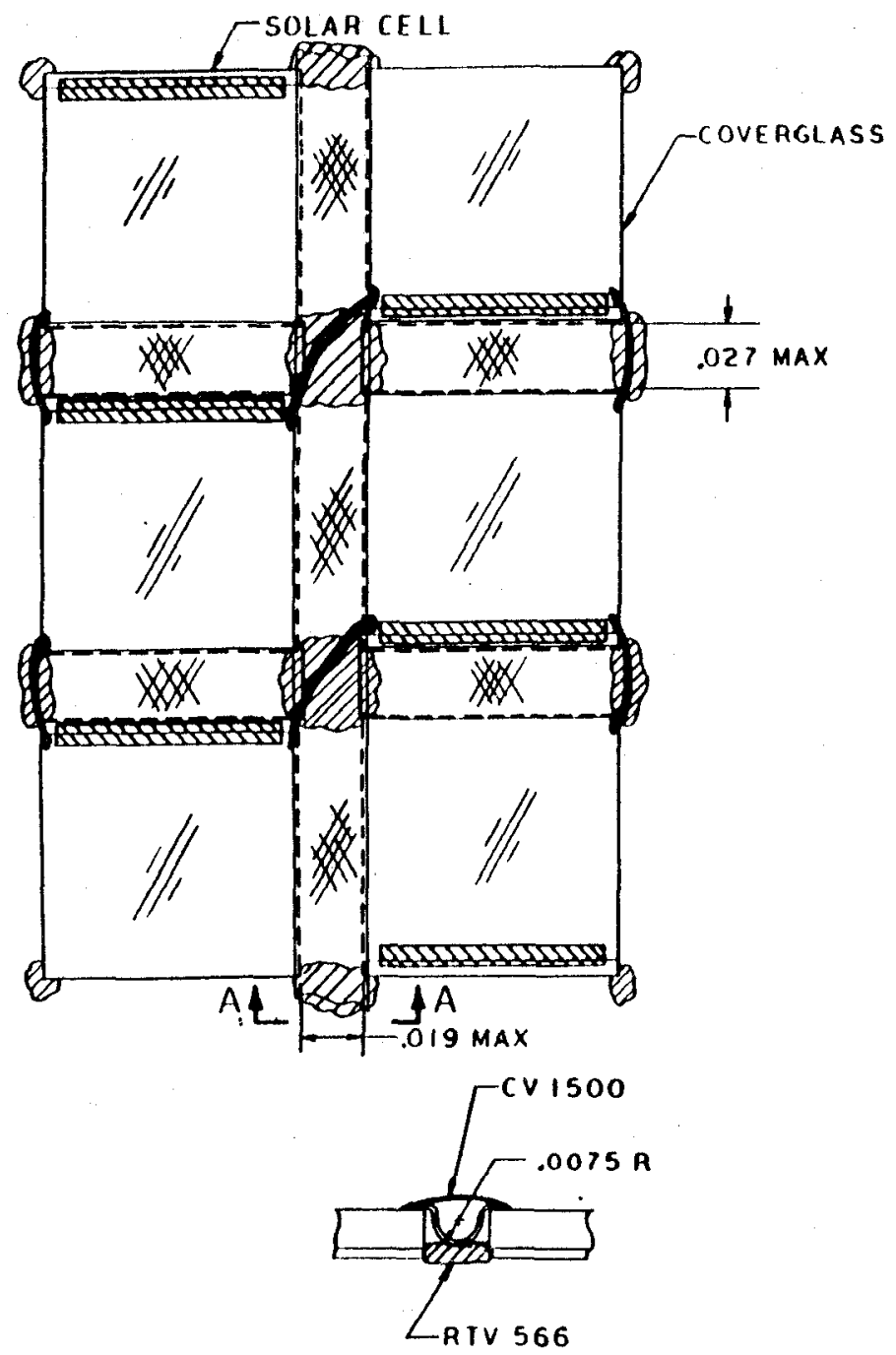

VIEW A-A

Fig. 1b. GGS2. Approach using silver mesh bonded to ITO coating with conductive silicon. 


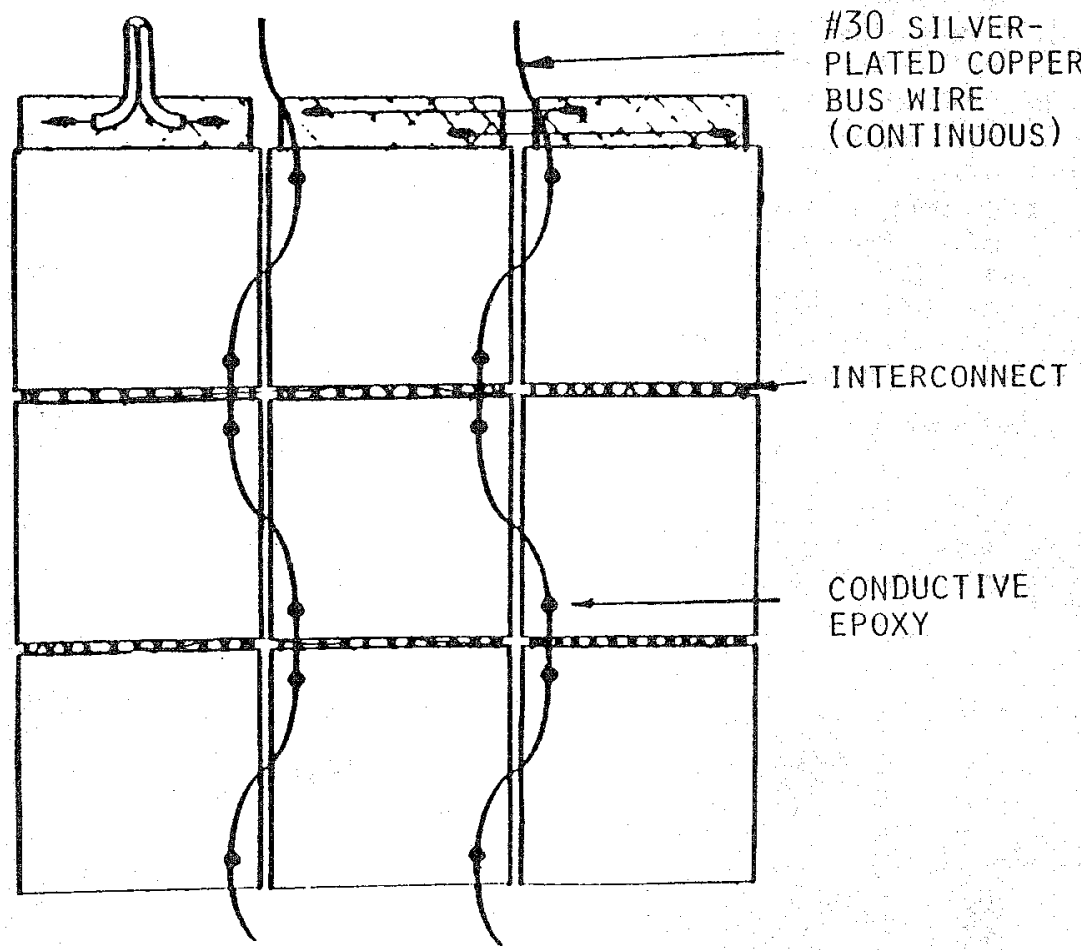

Fig. 1c. GGS3. Approach using silver wire epoxied to ITO coating .

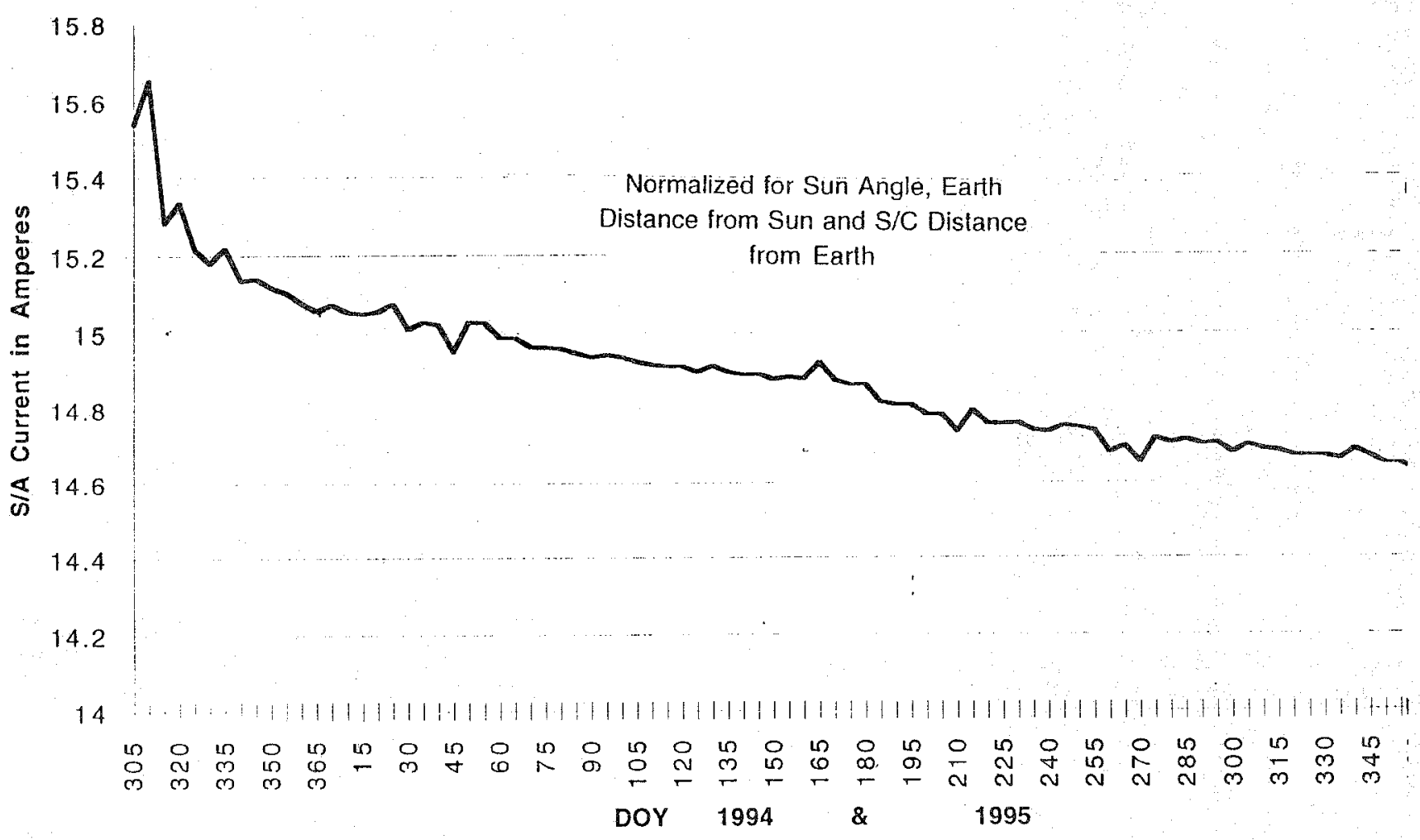

Fig. 2. 1 yr. WIND array degradation. 